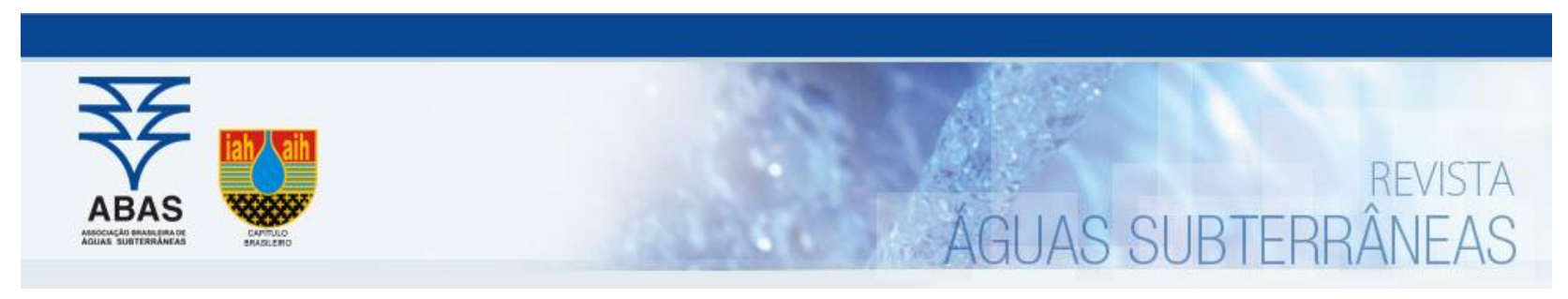

Artigos

\title{
Potencial biorremediador da microalga Chlorella vulgaris BEIJERINCK em meio composto por águas residuárias
}

\section{Bioremediator potential of the microalgae Chlorella vulgaris BEIJERINCK in a wastewater composed medium}

\author{
Djalma Queiroga de Assis Neto; Thiago Santos de Almeida Lopes ${ }^{1}$; Whelton Brito dos Santos ${ }^{1}$; Weruska Brasileiro Ferreira2; \\ Vera Lucia Antunes de Lima ${ }^{1 凶}$
}

1 Universidade Federal de Campina Grande (UFCG), Campus I, Campina Grande, PB, Brasil

2 Universidade Estadual da Paraíba (UEPB), Campus I, Campina Grande, PB, Brasil.

$\bowtie$ djalmaqueiroganeto@gmail.com, thiago.s.16@hotmail.com, wheltonbrt@gmail.com, weruska brasileiro@yahoo.com.br, vera.antunes.ufcg@gmail.com

\begin{abstract}
Palavras-chave:
Resumo

Dessalinização;

Filtro anaeróbio;

Biorremediação;

DCCR.

A dessalinização de águas salobras subterrâneas é, em muitas localidades, a única solução para o abastecimento de água. Porém, o processo de dessalinização por membrana de osmose inversa produz um rejeito poluente e de difícil tratamento. Similarmente, o efluente produzido pelo tratamento de esgoto doméstico em filtro anaeróbio também necessita de um pós-tratamento para se adequar às normas de despejo em corpos hídricos. Assim, a biorremediação de águas residuárias, utilizando microalgas, surge como uma opção de tratamento desse rejeito. 0 objetivo deste trabalho foi avaliar e otimizar o potencial biorremediativo da espécie de microalga Chlorella vulgaris em meio composto por rejeito de dessalinização por osmose inversa e efluente de tratamento de esgoto doméstico em filtro anaeróbio. Na metodologia foi utilizado o planejamento experimental por delineamento composto central rotacio-

Keywords: nado (DCCR), tendo como variáveis independentes: $\mathrm{pH}$, fotoperíodo e proporção de efluentes. Os parâmetros analisados foram a Demanda Química de Oxigênio (DQO) e o Fósforo Total (PT). As taxas de remoção para a variável DQO

Microalgae;

Wastewater;

Bioremediation;

CCRD.

variaram entre 28,73 e $70,73 \%$ e para a variável PT as taxas ficaram entre 52,19 e $100 \%$. A otimização estatística dos modelos mostrou uma remoção teórica combinada de 69,08\% para DQ0 e 89,34 PT.

Revisão por pares.

Recebido em: 24/11/2021.

Abstract

The groundwater desalination is, in many locations, the only solution for water supply. However, the reverse osmosis membrane desalination process produces a polluting and difficult-to-treat waste. Similarly, the effluent produced by the treatment of domestic sewage in anaerobic filter also needs a post-treatment to meet the standards for discharge into water bodies. Thus, the bioremediation of wastewater, using microalgae, appears as an option for treating this waste. The objective of this study was to evaluate and optimize the bioremediative potential of the microalgae species Chlorella vulgaris in a medium composed of reverse osmosis desalination wastewater and domestic sewage treatment effluent by anaerobic filter and the standard medium of the species. In the methodology, the experimental design was used by central composite rotational design (CCRD), having as independent variables: $\mathrm{pH}$, photoperiod and effluent concentration. The parameters analyzed were Chemical Oxygen Demand (COD) and Total Phosphorus (TP). Removal rates for the COD variable ranged between 28.73 and $70.73 \%$ and for the TP variable the rates were between 52.19 and $100 \%$. The statistical optimization of the models showed a combined theoretical removal of $69.08 \%$ for COD and $89,34 \%$ TP.
\end{abstract}

DOI: http:/doi.org/10.14295/ras.v35i3.30095

\section{INTRODUÇÃO}

Em regiões áridas e semiáridas a oferta de água é insuficiente para o desenvolvimento das diversas atividades e, por muitas vezes, a população ali residente utiliza fontes de água subterrânea, as quais são comumente classificadas como salobras ou salinas (NEVES et al., 2017). Para fins de tratabilidade dessas águas, as tecnologias de dessalinização por membranas surgem como uma alternativa viável e já bastante difundida (SURWADE et al., 2015). Em algumas localidades, a dessalinização não é só uma fonte complementar de abastecimento, mas a principal. Em âmbito internacional, países como o Qatar e Kuwait são completamente abastecidos por água dessalinizada (GHAFFOUR et al., 2015; NEVES et al., 2017).

A tecnologia de dessalinização mais difundida é a separação por membrana, osmose inversa ou osmose reversa, com sistemas espalhados em diversos países que convivem com a escassez hídrica, tendo seu uso datado desde a década de 70 (MAZLAN et al., 2016). A sua popularidade é devida principalmente a sua simplicidade e ao consumo energético reduzido, quando comparado ao processo de dessalinização por destilação (MAZLAN et al., 2016; SHENVI et al., 2015). Porém, o processo de dessalinização por membranas de osmose in- 
versa produz um rejeito com alta concentração salina e de difícil tratamento e disposição final adequada, sendo esse um dos principais problemas relacionados a sua difusão em regiões não-litorâneas (SARMENTO et al., 2014; NEVES et al., 2017).

Outro tipo de água residuária que demanda atenção são os efluentes de tratamentos anaeróbios de esgotos domésticos. Dentre os tratamentos secundários anaeróbios, pode-se destacar os filtros anaeróbios, que são reatores biológicos formados por um tanque de pedras de diferentes granulometrias, servindo de base para retenção e desenvolvimento de microrganismos digestores. São percebidos como um sistema de tratamento satisfatório para efluentes com baixa concentração de sólidos suspensos (CAVALCANTE et al., 2010; BAETTKER et al., 2018). Em contraponto, CHERNICHARO et al. (2001) elencam algumas desvantagens dos sistemas anaeróbios, como remoção insatisfatória de nutrientes e necessidade de pós-tratamento devido, principalmente, a baixa estabilização de matéria orgânica.

Neste contexto, o tratamento de águas residuárias requer processos altamente onerosos, por possuir elevado acúmulo de matéria orgânica, uma ampla gama de sais inorgânicos em alta concentração, metais pesados e nutrientes(Vo et al., 2019). Diversas soluções para reduzir esta carga poluente vem sendo estudadas e uma destas é a biorremediação através de microalgas, atraindo cada vez mais interesse por ser uma alternativa de baixo custo e ecologicamente viável (VO et al., 2019). A utilização de microalgas na biorremediação de águas residuárias já é largamente estudada. Dentre estes estudos, alguns tratam de sua implementação em cultivo com rejeito de dessalinização (ANDREOTTI et al., 2017, AKBARZADEH e SHARIATI, 2014; DIAS et al., 2019; MATOS et al., 2018). Porém, devido à alta concentração de sais minerais, o rejeito oriundo de dessalinização é mais eficiente como meio de cultivo quando combinado com outros efluentes, uma vez que tal diluição reduz a concentração de salinidade (MATOS et al., 2017; ZHOU et al., 2017; SHIRAZI et al., 2018).

Assim, uma das espécies que tem ganhado atenção para cultivo em águas residuárias é a Chlorella vulgaris BEIJERINCK (1890). Sendo classificada como alga verde, filo Chlorophyta, classe Chlorophyceae, ordem Chlorococcales, família Oocystaceae e gênero Chlorella. São unicelulares de água doce, com amido como principal fonte de reserva e a celulose como principal componentes da parede celular (SANTOS, 2013). Possuem importantes características, como: Crescimento acelerado, adaptação aos meios de cultura flexíveis e de relativa facilidade de obtenção, além de possuir resistência a fatores externos. Além disso, já se encontram aplicações de sua biomassa na indústria alimentícia, na farmacêutica e na produção de biocombustível (DALIRY et al., 2017).

Portanto, o foco deste trabalho foi avaliar e otimizar a capacidade biorremediadora de um meio contendo efluente doméstico tratado por filtro anaeróbio e rejeito de dessalinização proveniente do sistema de osmose inversa, através do cultivo da microalga Chlorella vulgaris.

\section{MATERIAL E MÉTODO}

A pesquisa foi desenvolvida no Laboratório de Referência em Tecnologias de Tratamento de Águas (LARTECA), da Universidade Estadual da Paraíba, UEPB, localizada no Município de Campina Grande-PB, coordenadas: Latitude -7.2074, Iongitude -35.9181 e altitude $550 \mathrm{~m}$.

Os cultivos tiveram duração de 9 dias e foram realizados em Erlenmeyres de $1000 \mathrm{~mL}$, com aclimatação em cepários à temperatura ambiente $\left(26 \pm 2{ }^{\circ} \mathrm{C}\right)$, iluminação por meio de lâmpadas fluorescentes com potência de $40 \mathrm{~W}$ e injeção direta de ar comprimido através de bomba de ar, para oxigenação e homogeneização dos cultivos. A Figura 1 apresenta o esquema do aparato experimental utilizado para a aclimação dos cultivos.

Figura 1 - Cultivos da microalga Chlorella vulgaris em escala de bancada

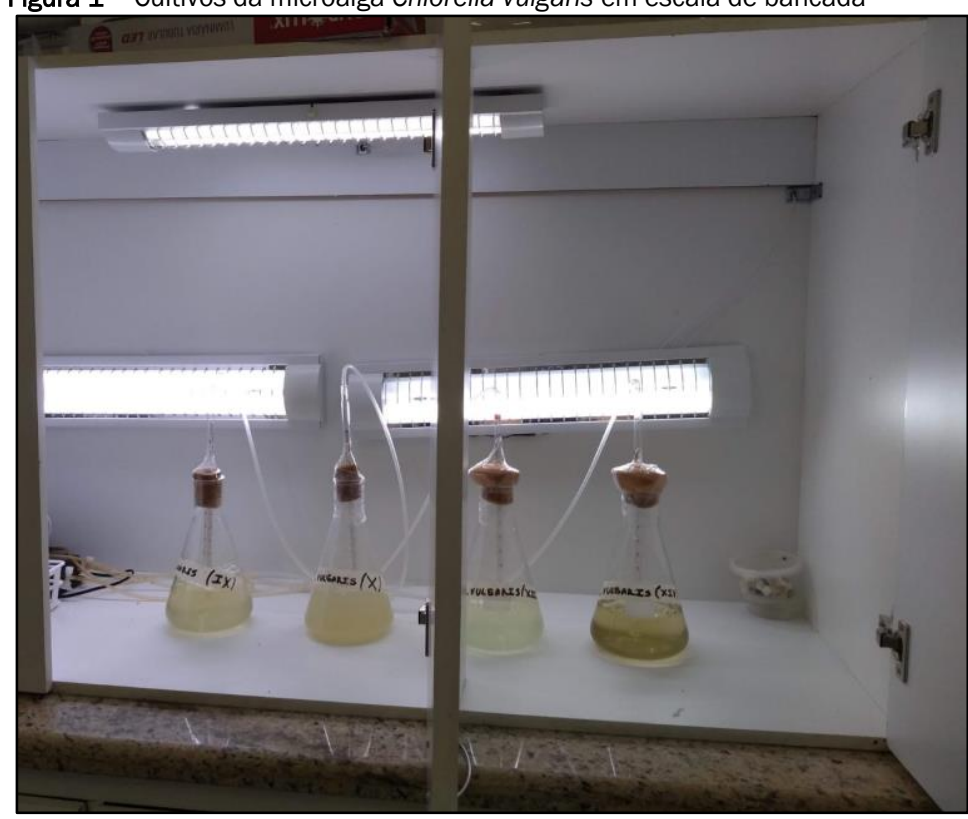

Fonte: Autoria Própria (2021). 
As parcelas utilizadas para compor o meio misto de cultivo foram: Rejeito de dessalinização, captado em uma planta de dessalinização de osmose inversa, situada no município de Juazeirinho, Paraíba; efluente de filtro anaeróbio proveniente do Instituto Nacional do Semiárido (INSA); E meio BBM, recomen- dado para o cultivo da Chlorella vulgaris (NICHOLS, 1973), modificado com adição de extrato de levedura e preparado no laboratório (LARTECA). Na Tabela 1 são apresentados os parâmetros de qualidade destas parcelas.

Tabela 1 - Parâmetros das parcelas que compõem o meio misto

\begin{tabular}{lcccc} 
Parâmetros & Unidade & $\begin{array}{c}\text { Rejeito de } \\
\text { dessalinização }\end{array}$ & $\begin{array}{c}\text { Efluente de filtro } \\
\text { anaeróbio }\end{array}$ & Meio Padrão BBM* \\
\hline Fósforo total (PT) & mg.L-1 & 0,09 & 5,30 & 28,90 \\
DQO & mg.L-1 & 277,78 & 116,40 & 297,62 \\
Salinidade & PSU & 10,55 & 0,44 & 0,36 \\
\hline * Bold's Basal Medium (NICHOLS, 1973).
\end{tabular}

Para o planejamento experimental e posterior análise de dados e otimização do modelo estatístico foi utilizado o delineamento composto central rotacionado (DCCR). Sendo um modelo de segunda ordem, representa de forma bem aproximada a interação entre os efeitos principais lineares e não-lineares, utilizando pontos axiais para estimativa de efeitos de segunda ordem (PAULA; FERNANDES, 2018).

Foram selecionados três fatores ou variáreis independentes: $\mathrm{pH}$, fotoperíodo e proporção de efluentes (PDE). A definição do limite baixo, alto e central do $\mathrm{pH}$ de cada fator foi feito com base nas faixas de melhor eficiência de desenvolvimento algal descrita pela literatura (ÇELEKLI; DÖNMEZ, 2006; BELTRÁNROCHA et al., 2017; DALIRY et al., 2017, KHALIL et al., 2010, GONG et al., 2014). Para as faixas de PDE foi fixado o meio
BBM em 50\% do volume total, enquanto os teores de rejeito de dessalinização e efluente de filtro anaeróbio variaram complementarmente entre 0 e 50\%. Por fim, na delimitação do fotoperíodo foi levado em conta a variação do tempo de luz solar médio anual do Brasil (ANEEL, 2020), objetivando uma aplicabilidade em larga escala com custos reduzidos. 0 planejamento experimental gerou 18 experimentos, sendo 4 pontos centrais. 0 mesmo pode ser observado com mais detalhes através da Tabela 2, que apresenta os valores codificados e não codificados dos níveis dos fatores selecionados (variáveis independentes).

A matriz de delineamento pode ser percebida na Tabela 3, apontando as características dos 18 experimentos gerados.

Tabela 2 - Fatores e níveis do Delineamento Composto Central Rotacionado (DCCR)

\begin{tabular}{|c|c|c|c|c|c|c|c|}
\hline \multirow{2}{*}{ Fatores } & \multirow{2}{*}{ Unidade } & \multirow{2}{*}{ Símbolo } & \multicolumn{5}{|c|}{ Níveis } \\
\hline & & & $-1,68$ & -1 & 0 & 1 & 1,68 \\
\hline $\mathrm{pH}$ & - & $X_{1}$ & 6,0 & 7,0 & 8,5 & 10,0 & 11 \\
\hline Fotoperíodo & $\mathrm{h}$ & $X_{2}$ & 8,6 & 10,0 & 12,0 & 14,0 & 15,4 \\
\hline PDE* & $\%$ & $X_{3}$ & $0,0: 50,0$ & $10,0: 40,0$ & $25,0: 25,0$ & $40,0: 10,0$ & $50,0: 0,0$ \\
\hline
\end{tabular}

* PDE (Proporção de efluentes) = \% Efluente de filtro anaeróbio : \% Rejeito de dessalinização

As variáveis dependentes utilizadas para indicar a eficiência do tratamento foram a demanda química de oxigênio (DQO) e fósforo total (PT), aferidas no início do cultivo e no fim, transcorridos os 9 dias de cultivo, então expressas em termos de remoção (\%). A determinação destes parâmetros foi feita pelas metodologias conforme Standard Methods for the Examination of Water and Wastewater (APHA; AWWA; WPCF, 2012).

Modelos de regressão linear e quadrática foram utilizados para explicar estatisticamente a relação entre as variáveis in- dependentes e as dependentes. A compatibilidade dos modelos foi testada através da análise de variância e a previsão das melhores condições de cultivo foi realizada pela função de desejabilidade, a qual buscou a combinação dos fatores de forma que todas as variáveis de resposta fossem maximizadas simultaneamente. A análise dos resultados de desejabilidade foi realizada com base na escala de Harrington (RIBARDO; ALLEN, 2003). Para todas as análises foi utilizado o software Statistica ${ }^{\circledR} 10.0$ (STATSOFT, 2011). 
Tabela 3 - Matriz de experimentos do Delineamento Composto Central Rotacionado (DCCR) com variáveis codificadas e decodificadas

\begin{tabular}{|c|c|c|c|c|c|c|}
\hline \multirow{3}{*}{$\frac{\text { Cultivo }}{1}$} & \multicolumn{6}{|c|}{ Variáveis independentes } \\
\hline & \multicolumn{2}{|c|}{$\mathrm{pH}$} & \multicolumn{2}{|c|}{ Fotoperíodo (h) } & \multicolumn{2}{|c|}{ PDE (\%:\%) } \\
\hline & -1 & 7 & -1 & 10 & -1 & $10,0: 40,0$ \\
\hline II & -1 & 7 & -1 & 10 & 1 & $40,0: 10,0$ \\
\hline III & -1 & 7 & 1 & 14 & -1 & $10,0: 40,0$ \\
\hline IV & -1 & 7 & 1 & 14 & 1 & $40,0: 10,0$ \\
\hline v & 1 & 10 & -1 & 10 & -1 & $10,0: 40,0$ \\
\hline $\mathrm{VI}$ & 1 & 10 & -1 & 10 & 1 & $40,0: 10,0$ \\
\hline VII & 1 & 10 & 1 & 14 & -1 & $10,0: 40,0$ \\
\hline VIII & 1 & 10 & 1 & 14 & 1 & $40,0: 10,0$ \\
\hline IX & $-1,68$ & 6 & 0 & 12 & 0 & $25,0: 25,0$ \\
\hline$x$ & 1,68 & 11 & 0 & 12 & 0 & $25,0: 25,0$ \\
\hline $\mathrm{XI}$ & 0 & 8,5 & $-1,68$ & 8,6 & 0 & $25,0: 25,0$ \\
\hline XII & 0 & 8,5 & 1,68 & 15,4 & 0 & $25,0: 25,0$ \\
\hline XIII & 0 & 8,5 & 0 & 12 & $-1,68$ & $0,0: 50,0$ \\
\hline XIV & 0 & 8,5 & 0 & 12 & 1,68 & $50,0: 0,0$ \\
\hline$x V$ & 0 & 8,5 & 0 & 12 & 0 & $25,0: 25,0$ \\
\hline $\mathrm{XVI}$ & 0 & 8,5 & 0 & 12 & 0 & $25,0: 25,0$ \\
\hline XVII & 0 & 8,5 & 0 & 12 & 0 & $25,0: 25,0$ \\
\hline XVIII & 0 & 8,5 & 0 & 12 & 0 & $25,0: 25,0$ \\
\hline
\end{tabular}

\section{RESULTADOS E DISCUSSÃO}

As melhores taxas de remoção para a variável DQO obtiveram uma média de $70,57 \%$ e para a variável PT, de $97,96 \%$.Os valores iniciais de DQO variaram entre 207 e 322 mg.L-1, para as diferentes configurações de cultivo e os valores finais ficaram entre 63 e 195 mg. L-1. Para a variável PT os valores iniciais oscilaram entre 13,6 e 17,1 mg.L-1 e os finais entre 0 (Não detectável) e 8,4 mg.L-1 (Tabela 4).

Também pode-se perceber pela Tabela 1 que a própria combinação dos efluentes para compor o meio dilui o potencial poluidor deles. A presença do rejeito de dessalinização pode reduzir a concentração de fósforo, pois apresenta um teor deste bem inferior aos demais (Tabela 1). 0 efluente de filtro anaeróbio também pode diminuir o valor conjunto de DQO e de salinidade (Tabela 1). Por fim, o meio padrão BBM também pode diluir a salinidade presente no rejeito de dessalinização, além de apresentar outros nutrientes complementares ao cultivo da espécie (Tabela 1).

Em estudo utilizando águas residuárias municipais como meio de cultivo da C. vulgaris, Chaudhary et al. (2020) obtiveram taxas de remoção de DQO máximas entre 74,4 e 85\%, moderadamente superior à máxima obtida neste estudo (70,73\%). Por sua vez, trabalho desenvolvido com meio composto por BBM e águas residuárias de indústria têxtil, Subashini e Rajiv (2018) alcançaram taxas de até $85 \%$. Em relação ao fósforo, Zhou et al. (2014), em cultivos com a C. vulgaris em águas residuárias municipais, registrou redução de $82,2 \%$ de PT. Outros estudos, com cultivos similares, estudaram remoção de fosfatos, apresentando reduções entre 39,14 e 87,3\% (SUBASHINI; RAJIV, 2018; AJALA; ALEXANDER, 2019), valores eficientes, mas ainda assim inferiores ao valor máximo obtido no presente trabalho (100\%). Estes estudos trazem resultados que reforçam a aplicabilidade da C. vulgaris para biorremediação de efluentes, pois em sua grande maioria, conseguiram reduzir mais da metade dos teores de DQO e PT. 
Tabela 4 - Resultados de remoção de Demanda Química de Oxigênio (DQO) e Fósforo Total (PT) para os experimentos realizados a partir da matriz de ensaios obtidos pelo planejamento em DCCR

\begin{tabular}{|c|c|c|c|c|c|c|}
\hline \multirow{3}{*}{$\begin{array}{c}\text { Cultivo } \\
1\end{array}$} & \multicolumn{6}{|c|}{ Variáveis dependentes } \\
\hline & \multicolumn{3}{|c|}{ DQO (\%) } & \multicolumn{3}{|c|}{ PT (\%) } \\
\hline & 28,86 & \pm & 4,62 & 82,68 & \pm & 0,54 \\
\hline II & 38,77 & \pm & 4,27 & 52,19 & \pm & 1,09 \\
\hline III & 41,55 & \pm & 1,83 & 85,70 & \pm & 0,15 \\
\hline IV & 49,59 & \pm & 3,72 & 55,00 & \pm & 0,50 \\
\hline v & 47,48 & \pm & 3,33 & 90,22 & \pm & 0,39 \\
\hline VI & 66,83 & \pm & 3,35 & 64,04 & \pm & 0,61 \\
\hline VII & 52,15 & \pm & 4,38 & 88,26 & \pm & 0,15 \\
\hline VIII & 70,41 & \pm & 3,19 & 65,04 & \pm & 0,14 \\
\hline $\mathrm{IX}$ & 60,32 & \pm & 6,45 & 95,38 & \pm & 1,12 \\
\hline$x$ & 50,74 & \pm & 1,93 & 95,92 & \pm & 0,29 \\
\hline$X I$ & 41,59 & \pm & 3,11 & 86,32 & \pm & 2,60 \\
\hline XII & 60,5 & \pm & 4,27 & 81,81 & \pm & 1,07 \\
\hline XIII & 28,73 & \pm & 3,03 & 100,0 & \pm & 0,20 \\
\hline XIV & 70,73 & \pm & 2,91 & 66,68 & \pm & 1,08 \\
\hline$X V$ & 53,27 & \pm & 4,94 & 67,43 & \pm & 0,00 \\
\hline$X V I$ & 51,94 & \pm & 4,71 & 64,65 & \pm & 0,00 \\
\hline XVII & 53,45 & \pm & 2,80 & 63,72 & \pm & 0,00 \\
\hline XVIII & 54,86 & \pm & 4,15 & 61,87 & \pm & 0,00 \\
\hline
\end{tabular}

A Figura 2 apresenta os gráficos de Pareto para as variáveis DQO e PT, respectivamente, com significância estatística de $5 \%(p=0,05)$. Os gráficos de Pareto servem para mensurar a significância das estimativas dos efeitos principais e de segunda ordem dos fatores em relação às variáveis independentes, sendo a linha vertical a delimitação do ponto de significância e as variáveis que a cruzam são consideradas estatisticamente significantes (STATSOFT, 2011).

Tratando-se da redução de DQO (Figura 2a), os termos lineares de $\mathrm{PDE}$, fotoperíodo e $\mathrm{pH}$ têm forte influência na eficiência do tratamento, visto que apresentam valores superiores à linha de significância. A interação do efeito linear do pH com os outros dois lineares apresentam influência, como também os termos quadráticos de PDE e fotoperíodo. Por sua vez, na análise do gráfico de Pareto para remoção de PT (Figura 2b), o termo linear de PDE e o quadrático de $\mathrm{pH}$ apresentam forte influência. Também possuem significância o termo quadrático de fotoperíodo e o linear do $\mathrm{pH}$.

Baseando-se nos gráficos de Pareto (Figuras 2a e 2b), os termos não significantes foram retirados para um melhor ajuste dos modelos, com exceção do termo linear de fotoperíodo para a remoção de fósforo, pois apesar de apresentar baixa significância ( $p>0,05)$, o termo foi significante na regressão. A Tabela 5 apresenta os parâmetros estatísticos utilizados para avaliar a qualidade do ajuste dos modelos. 
Figura 2 - Gráficos de Pareto para taxa de redução de Demanda Química de Oxigênio (DQO) (a) e Fósforo Total (PT) (b)

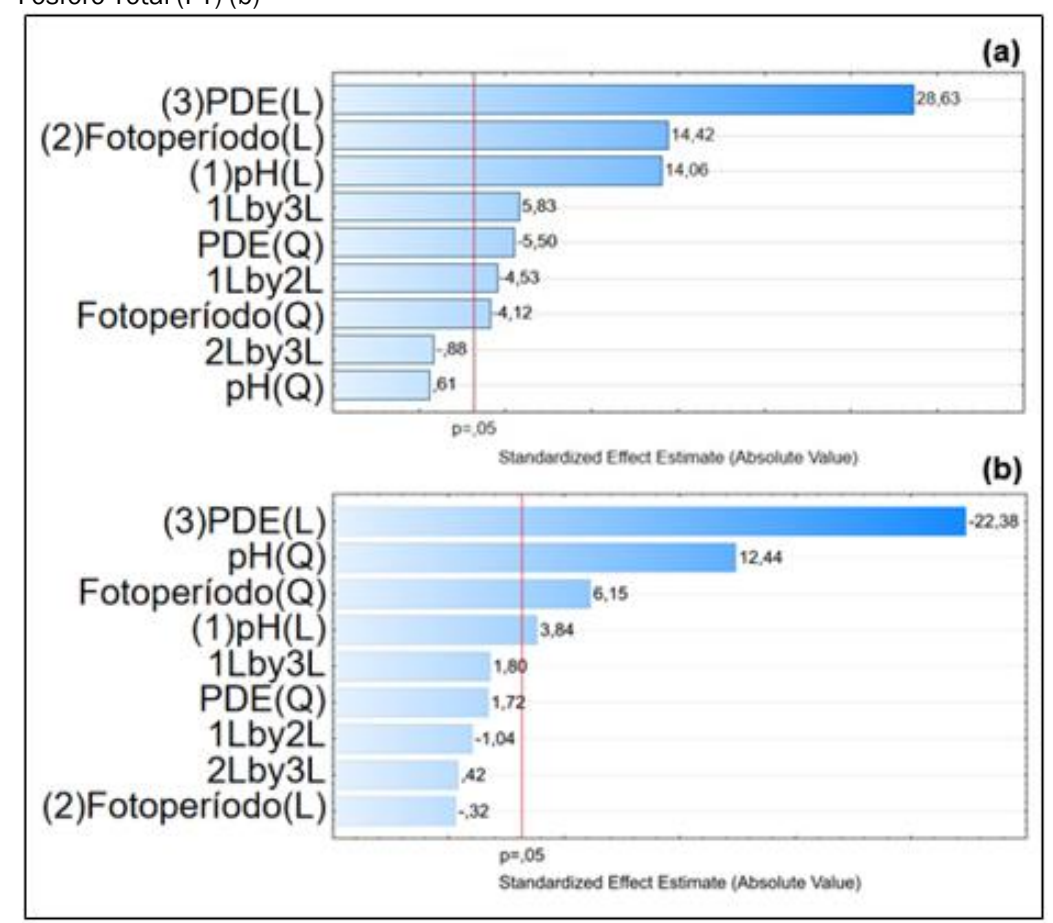

Fonte: Autoria Própria (Statistica 7), 2021.

Tabela 5 - Valores da análise de variância para o ajuste dos modelos estatísticos das variáveis Demanda Química de Oxigênio (DQO) e Fósforo Total (PT)

\begin{tabular}{lcc}
\multicolumn{1}{c}{ Estatística dos modelos } & DQO & PT \\
\hline $\mathrm{R}^{2}(\%)$ & 73,92 & 83,83 \\
$\mathrm{R}^{2}$ ajustado (\%) & 55,66 & 77,09 \\
$\mathrm{~F}_{\text {calc }} / \mathrm{F}_{\text {tab }}$ (regressão) & 1,29 & 4,01 \\
$\mathrm{~F}_{\text {calc }} / \mathrm{F}_{\text {tab }}$ (falta de ajuste) & 7,46 & 1,62 \\
\hline
\end{tabular}

Com base na Tabela 5, todos os modelos foram significativos, com nível de confiança de 95\%, Fcal/Ftab>1 (BARROS NETO et al., 2007). Porém, a falta de ajuste se mostrou significativa (Fcal/Ftab>1), dessa forma, os modelos não podem ser considerados preditivos, pois para que isso ocorra Fcal/Ftab $<1$ (BARROS NETO et al., 2007).

Todavia, por serem significativos, os modelos são eficazes para otimizar as variáveis dentro dos limites estudados, para ambas as remoções de DQO e PT, isto por ser um modelo significativo significa que os valores obtidos pelo modelo pos- suem efetiva semelhança com os experimentais, dentro dos limites. Além disso, sabe-se que o $\mathrm{R}^{2}$ indica o quanto os dados encontrados são explicados pelos modelos e que quanto mais próximo a $1(100 \%)$ for o $\mathrm{R}^{2}$ melhor será o modelo. Assim, os valores encontrados foram considerados bons, visto se tratar de uma pesquisa envolvendo microalgas, que são seres sensíveis a alterações ambientais-(DARVEHEl et al., 2018; GOLDMAN; RYTHER, 1976).

As Equações 1 e 2 apresentam os modelos de regressão para taxa de remoção de DQO e PT, respectivamente.

$$
\begin{aligned}
& R_{\text {DQO }}=-0,36 X_{2}^{2}-0,008 X_{3}^{2}+7,93 X_{1}+16,28 X_{2}+0,11 X_{3}-0,64 X_{1} X_{2}+0,11 X_{1} X_{3}-113,52 \\
& R_{P T}=3,49 X_{1}^{2}+0,94 X_{2}^{2}-57,83 X_{1}-22,74 X_{2}-0,94 X_{3}+465,67
\end{aligned}
$$

Onde, RDQO e RPT correspondem, respectivamente, a remoção (\%) de $\mathrm{DQO}$ e de fósforo total, $\mathrm{X}_{1}$ corresponde ao $\mathrm{pH}$, adimensional; $X_{2}$ é o Fotoperíodo, em horas (h) e $X_{3}$ o PDE (\% do efluente de filtro anaeróbio, sendo a do rejeito de dessalinização o valor complementar para $50 \%$ ).
A partir dos modelos estatísticos foram construídas as super fícies de resposta para as taxas de remoção de DQO e PT. As superfícies de resposta podem ser visualizadas na Figura 3. 
Figura 3 - Superfícies de respostas das remoções de Demanda Química de Oxigênio (DQO) e Fósforo Total (PT) em função das variáveis independentes $\mathrm{pH}$, fotoperíodo e proporção de efluentes (PDE)

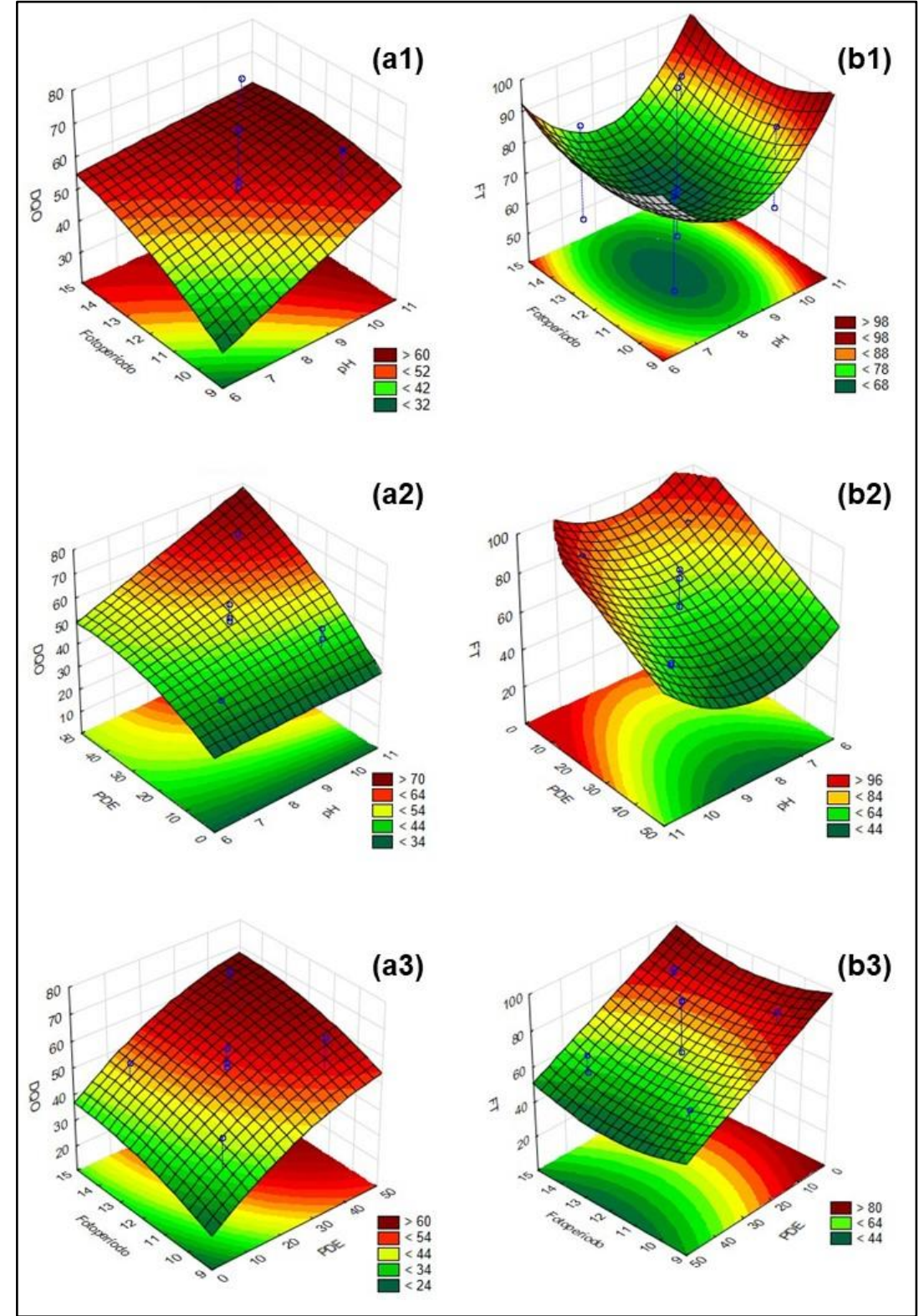

Fonte: Autoria Própria (Statistica 7), 2021

Ao manter a PDE no ponto médio (25\%:25\%) e variando o Fotoperíodo e o $\mathrm{pH}$, nota-se que as maiores remoções de DQO (Figura 3a1) ocorrem em fotoperíodo maior que 12 horas e $\mathrm{pH}$ maior que 9. Por sua vez, as maiores remoções de PT (Figura 3b1) foram observados próximos aos niveis extremos, tanto superiores quanto os inferiores.

Fixando o fotoperíodo no ponto central (12h) e correlacionando o pH e a PDE, a remoção de DQO (Figura 3a2) foi potencializada em maiores valores de $\mathrm{pH}$ com maiores valores de PDE (maior porcentagem de efluente de filtro anaeróbio e menor rejeito de dessalinização). Para o PT (Figura 3b2), maiores remoções foram observadas em maiores valores de PDE em ampla faixa de $\mathrm{pH}$.

Confrontando as variáveis PDE e fotoperíodo, mantendo-se o $\mathrm{pH}$ no ponto central $(8,5)$, notou-se que maiores fotoperíodos e PDE resultaram em maiores remoções de DQO (Figura 3a3). Porém, em relação ao PT (Figura 3b3) o fotoperíodo teve pouca influência, sendo observado maiores remoções em maiores niveis de PDE.

Uma importante característica da $D$. Tertiolecta é sua elevada tolerância a altos níveis de salinidade, suportando uma ampla gama de meios de cultivos salinos, mostrando assim o motivo de sua adaptação a diferentes PDEs, também possuindo o importante aspecto de suportar diferentes teores de luminosidade (BINTE SAFIE et al., 2018).

Isso possui coerência pois a luz é um dos fatores mais importantes na produtividade de microalgas, propiciando a energia requerida para as reações fotossintéticas ocorrerem, podendo desta forma converter os nutrientes orgânicos em biomassa. 
A eficiência de remoção de nutrientes por microalgas está diretamente ligada a intensidade de luz e ao fotoperíodo de exposição (SUTHERLAND et al., 2015; Ll et al., 2019).

Em todos os casos em que a PDE foi confrontada com as demais variáveis (Figuras 3a2, 3b2, 3a3 e 3b3), foi percebido que a PDE possuiu correlação direta com as remoções de DQO e PT, indicando resultados mais satisfatórios em maiores concentrações de efluente de filtro anaeróbio e, consequentemente, menores concentrações de rejeito de dessalinização. ZIGANSHINA et al (2021) estudaram o cultivo da espécie Chlorella sorokiniana em efluente anaeróbio diluído em BBM, chegando a uma remoção máxima de $85 \%$ de fosfato, afirmando também, que para melhores eficiências o teor de nutrientes deve ser controlado. Já Matos et al. (2014), ao cultivarem C. vulgaris em BBM diluído em até 30\% de rejeito de dessalinização, observaram que os melhores resultados de crescimento celular e de biomassa foram a baixos teores de rejeito (10\%). Assim, é percebido que a associação dos efluentes beneficia o cultivo.

A partir dos modelos ajustados, foi aplicada a função desejabilidade, para a obtenção da condição ótima das variáveis independentes para se obter a máxima remoção de DQO e PT por meio do tratamento com Chlorella vulgaris. A Figura 4 ilustra o perfil dos valores preditos das variáveis $\mathrm{pH}$, fotoperíodo e PDE.

Figura 4 - Perfil dos valores preditos e da desejabilidade para as variáveis Demanda Química de Oxigênio (DQO) e Fósforo Total (PT)

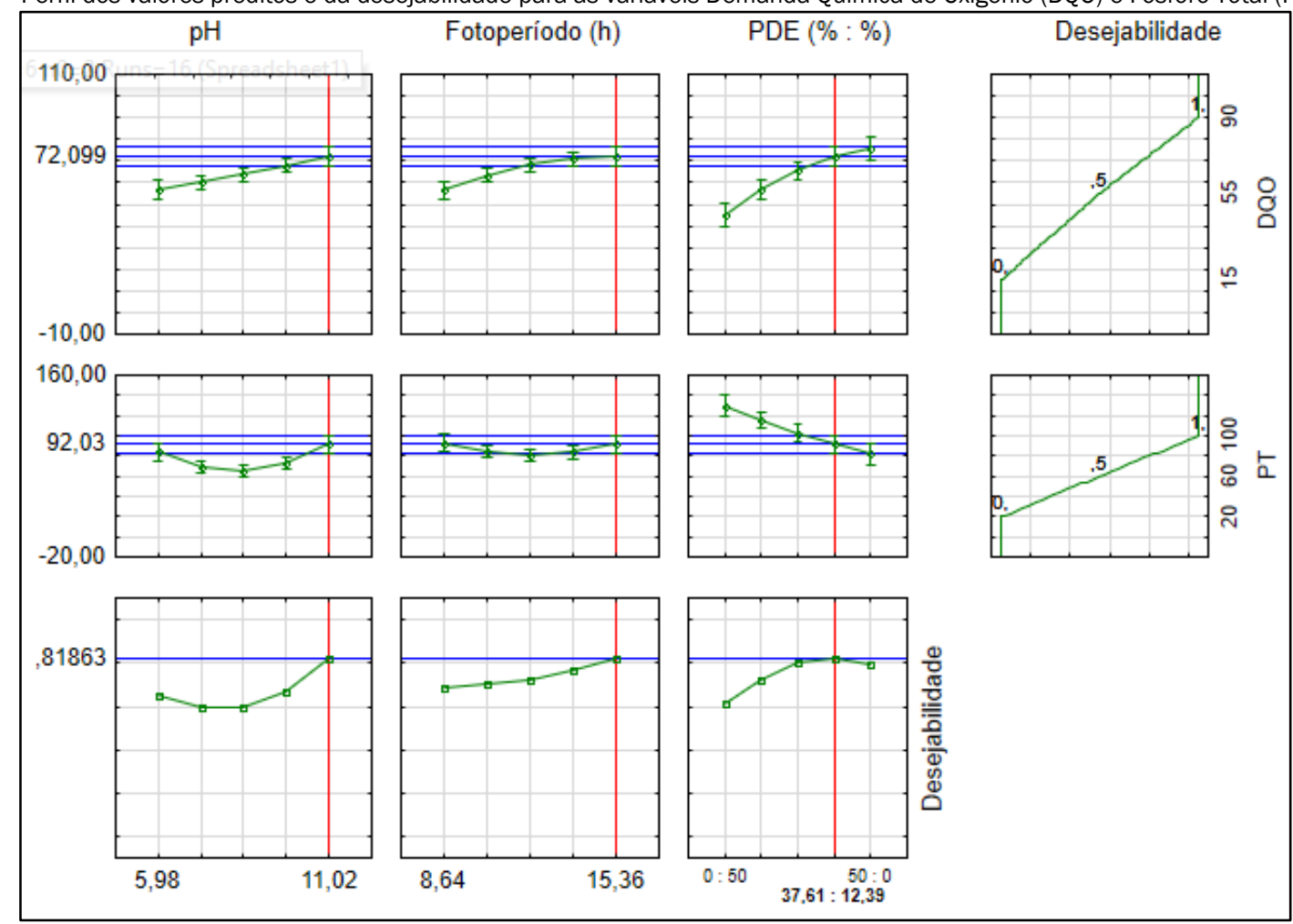

Fonte: Autoria Própria (Statistica 7), 2021.

Conforme os resultados de otimização obtidos a partir da desejabilidade, Figura 4, para se obter a maximização da remoção de DQO e PT de 72,099 e 92,035\%, respectivamente, é necessário o cultivo da Chlorella vulgaris em pH 11,023, fotoperíodo de 15,364 (h) e PDE de 37,61:12,39 (\%:\%). Sua otimização a maiores teores do efluente de filtro anaeróbio que de rejeito de dessalinização é esclarecida por ser uma espécie de água doce, além disso, a elevada presença de nutrientes no efluente de filtro anaeróbio favorece o crescimento algal (SALAMA et al., 2017). Ajala e Alexander (2019) afirmam que alguns mecanismos de remoção de nutrientes por microalgas acontecem à altos valores de $\mathrm{pH}$ (9-11). E, devido a C.vulgaris ser uma espécie de água doce, é mais sensível a valores elevados de salinidade.

Desta forma, percebe-se que a espécie apresenta mais eficiência para as variáveis em estudo quando cultivadas em meio básico, em períodos prolongados de exposição à luz e maior teor de efluente de filtro anaeróbio que de rejeito de dessalinização.

Por seu turno, a desejabilidade global resultante foi de 0,81863 . De acordo com a escala de Harrington (HARRINGTON, 1965), esse valor é classificado como aceitável e excelente (qualidade incomum ou desempenho bem além do que está disponível no mercado) (RIBARDO; ALLEN, 2003).

A partir dos valores otimizados e aplicando-os nos modelos obtidos (Equações 1 e 2), foram obtidos valores de remoções teóricos de 69,08\% para DQ0 e 89,34\% para PT, valores de ótimo desempenho quando comparados com outros estudos da literatura (SUBASHINI; RAJIV, 2018; AJALA; ALEXANDER, 2019; CHAUDHARY et al., 2020; ZHOU et al.,2014). 
Assim, levando em conta as taxas de remoção teóricas encontradas e os valores otimizados das variáveis dependentes, pôde-se chegar a valores finais teóricos, utilizando como os iniciais a ponderação dos valores da caracterização dos efluentes (Tabela 1). Esses valores podem ser observados na Tabela 6 .

Tabela 6 - Valores iniciais e finais teóricos após otimização

\begin{tabular}{|c|c|c|}
\hline & \multicolumn{2}{|c|}{ Parâmetros } \\
\hline & DQO & Fósforo total (PT) \\
\hline $\mathrm{BBM}(\mathrm{mg} / \mathrm{L}-50 \%)$ & 148,81 & 14,45 \\
\hline Efluente de filtro anaeróbio (mg/L - 37,61\%) & 43,78 & 1,99 \\
\hline Rejeito de dessalinização (mg/L - 12,39\%) & 34,42 & 0,011 \\
\hline Valor inicial teórico (mg/L) & 227,01 & 16,45 \\
\hline Taxa de remoção ótima (\%) & 69,08 & 89,34 \\
\hline Valor final teórico (mg/L) & 70,19 & 1,75 \\
\hline
\end{tabular}

Observando a Tabela 6, os valores finais ótimos são 70,19 e $1,75 \mathrm{mg} / \mathrm{L}$, para remoções de DQO e fósforo total, respectivamente. A resolução federal CONAMA $N^{\circ} 430$, de 13 de maio de 2011 aponta que os limites de Fósforo Total poderão ser definidos por órgãos ambientais competentes, não apresentando valores próprios, como também não define limites de DQO para lançamento de efluentes.

Nesse sentido, a resolução CONSEMA No 355 de 13 de julho de 2017, do Rio Grande do Sul, revela-se bastante abrangente. Ela cita um limite de DQO para despejo de efluentes em corpo hídrico com valor máximo variando entre 150 e 330 mg. $\mathrm{L}^{-1}$, dependendo da sua vazão, assim o valor final ótimo $(70,19 \mathrm{mg} / \mathrm{L})$ atende os requisitos para qualquer faixa de vazão, com folga.

De forma semelhante, para fósforo total, a resolução menciona que em trechos com registro de floração de cianobactérias e onde ocorra a captação para abastecimento público deve haver controle do fósforo total e estabelece limites máximos para diferentes vazões de efluentes, ficando entre 1 e 4 mg. L-1. Neste caso o valor final otimizado $\left(1,75 \mathrm{mg} . \mathrm{L}^{-1}\right)$ obedece a 3 das 4 faixas de vazão, ficando somente acima do valor estabelecido para vazões acima de $10000 \mathrm{~m}^{3} \cdot \mathrm{d}^{-1}$ (CONSEMA, 2017).

A normativa do Equador (MINISTERIO DEL AMBIENTE, 2003) traz limites máximos para despejo em corpos de água doce de 250 mg.L-1para DQO e 10 mg/L para Fósforo Total. A outro exemplo internacional, a legislação da Irlanda define tais limites em 125 e 2 mg.L-1para DQO e Fósforo Total, respectivamente (SCHELLENBERG et al., 2020).

Ademais, a biomassa resultante dos cultivos pode ser empregada em diversas vertentes econômicas, como a produção de biocombustíveis,sendo considerada de alta eficiência e prevista como uma fonte ambientalmente e economicamente sustentável para obtenção de derivados, possuindo também uma alta taxa de conversão energética (SUGANYA et al., 2016).

\section{CONCLUSÕES}

A utilização da microalga Chlorella vulgaris para biorremediação de efluentes se mostrou eficaz, apresentando elevadas taxas de remoção da demanda química de oxigênio e do fósforo total, sendo capaz de tornar os efluentes apropriados para lançamento, segundo a resolução CONSEMA № 355 de 13 de julho de 2017.

A aplicação do planejamento experimental DCCR, de modelos estatísticos e da função desejabilidade foram essenciais para chegar aos valores ótimos das variáveis em estudo.

A combinação do rejeito de dessalinização, do efluente de filtro anaeróbio e do meio BBM se revelou eficiente para o desenvolvimento algal, onde a proporção de efluentes mostrouse uma variável de grande influência na capacidade biorremediativa da espécie em relação a demanda química de oxigênio e ao fósforo total. Além disso, a diluição do rejeito de dessalinização reduz seu teor de salinidade, mitigando os impactos ambientais relacionados a este poluente.

\section{REFERÊNCIAS}

AJALA, S. O.; ALEXANDER, M. L. Assessment of Chlorella vulgaris, Scenedesmus obliquus, and Oocystis minuta for removal of sulfate, nitrate, and phosphate in wastewater. International Journal of Energy and Environmental Engineering, 2019. https://doi.org/10.1007/s40095-019$\underline{00333-0}$

AKBARZADEH, N.; SHARIATI, M. Aluminum remediation from medium by Dunaliella. Ecological Engineering v. 67, p. 76-79, 2014. https://doi.org/10.1016/i.ecoleng.2014.03.014

ANDREOTTI V.; et al. Bioremediation of aquaculture wastewater from Mugil cephalus (Linnaeus, 1758) with different microalgae species. Chemistry and Ecology, v.37, p.750-761, 2017.

https://doi.org/10.1080/02757540.2017.1378351

ANEEL - Agência Nacional de Energia Elétrica. Tabela 3.1: Duração solar do dia, em horas, em diferentes latitudes e períodos do ano. Atlas de Energia Solar. Disponível em: 
http://www2.aneel.gov.br/aplicacoes/atlas/energia_solar/3 2.htm. 2020. Acesso em: 12 Jun. 2021.

APHA; AWWA; WPCF. Standard Methods for the Examinaton of Water and Wastewater. 22. ed. Washington, D.C.: American Public Health Associaton/American Water Works Associaton/Water Environment Federaton, 2012.

BAETTKER, E. C.; CARVALHO, K. Q.; FREIRE, F. B.; PASSIG, F. H.; MORAIS, J. L. Materiais alternativos como meio suporte de filtros anaeróbios para tratamento de esgoto sanitário sintético. Revista Engenharia Sanitária e Ambiental, v. 23, n. 6, p. 1091-1102, 2018. https://doi.org/10.1590/s1413$\underline{41522018170758}$

BARROS NETO, B.; et al. Como fazer experimentos: pesquisa e desenvolvimento na ciência e na indústria. 3. ed. Campinas: UNICAMP, 2007.

BELTRÁN-ROCHA, C. J.; et al. Biotratamiento de efluentes secundarios municipales utilizando microalgas: Efecto del pH, nutrientes ( $\mathrm{C}, \mathrm{N}$ y $\mathrm{P}$ ) y enriquecimiento con $\mathrm{CO} 2$. Revista de Biología Marina y Oceanografía, v. 52, n. 3, p. 417-427, 2017. https://doi.org/10.4067/S0718-19572017000300001

BINTE SAFIE, S. R.; NG, Y. K.; YAO, L.; LEE, Y. K. Growth bottlenecks of microalga Dunaliella tertiolecta in response to an up-shift in light intensity. European Journal of Phycology, v. 53, n. 4, p. 509-519, 2018.

https://doi.org/10.1080/09670262.2018.1478131

CAVALCANTE, F. L.; ANDRADE NETO, C. O. DE; ARAÚJO, A. L. C.; MELO, H. N. DE S. Eficiência sanitária de filtros anaeróbios avaliada em função da remoção de ovos de vermes e coliformes fecais. Revista AIDIS, v. 3, n. 1, 2010.

ÇELEKLI, A. DÖNMEZ, G. Effect of pH, light intensity, salt and nitrogen concentrations on growth and b-carotene accumulation by a new isolate of Dunaliella sp. World Journal of Microbiology \& Biotechnology, v. 22, p. 183-189, 2006. https://doi.org/10.1007/s11274-005-9017-0

CHAUDHARY, R. et al. Kinetic study of nutrients removal from municipal wastewater by Chlorella vulgaris in photobioreactor supplied with CO2-enriched air. Environmental Technology, v. 41, n. 5, p. 617-626, 2020.

https://doi.org/10.1080/09593330.2018.1508250

CHERNICHARO, C. A. DE L.; VAN HAANDEL, A.; AISSE, M. M.; CAVALCANTI, P. F. F. Reatores Anaeróbios de Manta de Lodo. Tratamento de Esgotos Sanitários Por Processo Anaeróbio e Disposição Controlada no Solo, 1999. Rio de Janeiro-RJ: FINEP/PROSAB.

DALIRY, S.; et al. Investigation of optimal condition for Chlorella vulgaris microalgae growth. Global Journal of Environmental Science and Management, 2017.

DARVEHEI, P.; BAHRI, P. A.; MOHEIMANI, N. R. Model development for the growth of microalgae: A review. Renewable and Sustainable Energy Reviews, v. 97, p. 233258, 2018. https://doi.org/10.1016/j.rser.2018.08.027

DIAS, G.; et al. Biorremediação de efluentes por meio da aplicação de microalgas: uma revisão. Química Nova, 2019. https://doi.org/10.21577/0100-4042.20170393
GHAFFOUR, N.; et al. Renewable energy-driven desalination technologies: A comprehensive review on challenges and potential applications of integrated systems. Desalination, 5. jan. 2015. https://doi.org/10.1016/j.desal.2014.10.024

GOLDMAN, J. C.; RYTHER, J. H. Temperature-influenced species competition in mass cultures of marine phytoplankton. Biotechnology and Bioengineering, v.18, p. 1125-1144, 1976. https://doi.org/10.1002/bit.260180809

GONG, Q.; et al. Effects of light and pH on cell density of Chlorella vulgaria. Energy Procedia, v. 61, p. 2012-2015, 2014. https://doi.org/10.1016/j.egypro.2014.12.064

HARRINGTON, E. C. The desirability function. Industrial Quality Control, p. 494-498, Abril de 1965.

KHALIL, Z. I.; et al. A Effect of pH on growth and biochemical responses of Dunaliella bardawil and Chlorella ellipsoidea. World Journal of Microbiology and Biotechnology, v. 26, p. 1225-1231, 2010. https://doi.org/10.1007/s11274-009$\underline{0292-z}$

LI, K.; LIU, Q.; FANG, F.; et al. Microalgae-based wastewater treatment for nutrients recovery: A review. Bioresource Technology, v. 291, p. 121934, 2019.

https://doi.org/10.1016/i.biortech.2019.121934

MATOS, A. P.; MOECKE, E. H. S; SANT'ANNA, E. S. The use of desalination concentrate as a potential substrate for microalgae cultivation in Brazil. Algal Research, v. 24, p. 505508, 2017. https://doi.org/10.1016/i.algal.2016.08.003

MATOS, A. P.; et al. CULTIVATION OF Chlorella vulgaris IN MEDIUM SUPPLEMENTED WITH DESALINATION CONCENTRATE GROWN IN A PILOT-SCALE OPEN RACEWAY. Brazilian Journal of Chemical Engineering, v. 35, n. 4, p. 11831192, 2018.2 https://doi.org/10.1590/0104$\underline{6632.20180354 s 20170338}$

MAZLAN, N. M.; PESHEV, D.; LIVINGSTON, A. G. Energy consumption for desalination - A comparison of forward osmosis with reverse osmosis, and the potential for perfect membranes. Desalination, v. 377, p. 138-151, 2016. https://doi.org/10.1016/i.desal.2015.08.011

MINISTERIO DEL AMBIENTE. 31 de março de 2003. Norma de calidad ambiental y descarga de efluentes: recurso agua. Texto Unificado de Legislación Ambiental Secundaria del Ministerio del Ambiente. Quito, Ecuador. 2003.

NEVES, A. L. R.; et al. Aspectos socioambientais e qualidade da água de dessalinizadores nas comunidades rurais de Pentecoste-CE. Revista Ambiente e Agua, v. 12, n. 1, p. $124-$ 135, 2017. https://doi.org/10.4136/ambi-agua.1722

NICHOLS, H. W. Growth media freshwater. In: STEIN, J. (Ed.), Handbook of phycological methods: culture methods and growth measurements. Cambridge University Press, p. 7-24, 1973.

PAULA, H. M. de; FERNANDES, C. E. Otimização do tratamento de água cinza a partir do uso combinado de coagulantes químicos. Revista Engenharia Sanitária e Ambiental, v. 23, n. 5, 2018. https://doi.org/10.1590/s1413-41522018169155

RIBARDO, C.; ALLEN, T. T. An alternative desirability function for achieving "six sigma" quality. Quality and Reliability 
Engineering International, v. 19, n. 3, p. 227-240, 2003. https://doi.org/10.1002/are.523

RIO GRANDE DO SUL. Conselho Estadual do Meio Ambiente CONSEMA. Resolução $n^{\circ} 355$ de 13 de julho de 2017. Diário Oficial do Estado, Rio Grande do Sul, 2017.

SALAMA, E. S.; KURADE, M. B.; ABOU-SHANAB, R. A. I.; et al. Recent progress in microalgal biomass production coupled with wastewater treatment for biofuel generation. Renewable and Sustainable Energy Reviews, 2017. https://doi.org/10.1016/i.rser.2017.05.091

SANTOS, R. R. Estudo de diferentes condições de cultivo de chlorella vulgaris visando o aumento da produtividade em biomassa e lipídioS. Dissertação (Mestrado) - Programa de Pós-Graduação em Tecnologia de Processos Químicos e Bioquímicos, Universidade Federal do Rio de Janeiro - UFRJ. 2013.

SARMENTO, J. D. A.; et al. Qualidade e conservação da alface cultivada com rejeito da dessalinização. Revista Caatinga, v. 27, n. 3, p. 90-97, 2014.

SHENVI, S. S.; ISLOOR, A. M.; ISMAIL, A. F. A review on RO membrane technology: Developments and challenges. Desalination, 2015. https://doi.org/10.1016/i.desal.2014.12.042

SHIRAZI, S. A.; et al. Simultaneous biomass production and water desalination concentrate treatment by using microalgae. Desalination and Water Treatment, v. 135, p. 101-107, 2018. https://doi.org/10.5004/dwt.2018.23163

SCHELLENBERG, T.; SUBRAMANIAN, V.; GANESHAN, G.; TOMPKINS, D.; PRADEEP, R. Wastewater Discharge Standards in the Evolving Context of Urban Sustainability-The Case of India. Frontiers in Environmental Science, v. 8. Abril de 2020. https://doi.org/10.3389/fenvs.2020.00030

STATSOFT, I. STATISTICA (Data Analysis Software System), www.statsoft.com, versão 7, 2011.
SUBASHINI, P. S.; RAJIV, P. An Investigation of Textile Wastewater Treatment using Chlorella vulgaris. Oriental Journal Of Chemistry, v. 34, n. 5, p.2517-2524, 2018. https://doi.org/10.13005/ojc/340538

SUGANYA, T.; et al. Macroalgae and microalgae as a potential source for commercial applications along with biofuels production: A biorefinery approach. Renewable and Sustainable Energy Reviews, 2016. https://doi.org/10.1016/j.rser.2015.11.026

SURWADE, S. P.; et al. Water desalination using nanoporous single-layer graphene. Nature Nanotechnology, v. 10, n. 5, p. 459-464, 2015. https://doi.org/10.1038/nnano.2015.37

SUTHERLAND, D. L.; HOWARD-WILLIAMS, C.; TURNBULL, M. H.; BROADY, P. A.; CRAGGS, R. J. The effects of $\mathrm{CO} 2$ addition along a $\mathrm{pH}$ gradient on wastewater microalgal photo-physiology, biomass production and nutrient removal. Water Research, v. 70, p. 9-26, 2015 .

https://doi.org/10.1016/i.watres.2014.10.064

VO, H. N. P.; et al. Microalgae for saline wastewater treatment: a critical review. Critical Reviews in Environmental Science and Technology, 2019.

https://doi.org/10.1080/10643389.2019.1656510

ZHOU, G. et al. Simultaneous removal of inorganic and organic compounds in wastewater by freshwater green microalgae. Environmental Science: Processes and Impacts, v. 16, 2014. https://doi.org/10.1039/C4EM00094C

ZHOU, W.; et al. Nutrients removal and recovery from saline wastewater by Spirulina platensis. Bioresource Technology, v. 245 , p. 10-17, 2017. https://doi.org/10.1016/j.biortech.2017.08.160

ZIGANSHINA, E. E.; BULYNINA, S. S.; ZIGANSHIN, A. M. Assessment of Chlorella sorokiniana Growth in Anaerobic Digester Effluent. Plants, v. 10, p. 478, 2021. https://doi.org/10.3390/plants10030478 